\section{Spotlight on membrane traffic}

Nature Cell Biology, and indeed all other Nature Publishing Group journals, engage in special projects - Focuses, Supplements, Insights and Milestones — that are intended to highlight a journal's continuing commitment to areas of exceptional scientific interest. In this month's issue - a Focus on membrane traffic — we are pleased to present a series of specially commissioned articles by leading experts in the field.

Understanding how proteins are targeted to organelles or for secretion, and how they travel between intracellular compartments, has been an area of intense investigation since cell biology evolved as a discipline. From the largely phenomenological investigations of the 1960s, the field has moved to bolster initial morphological observations with a molecular and structural understanding of the underlying processes. This progression is summarized in a Historical Perspective article by Randy Schekman on page 483. As the field has matured, several fundamental issues have been resolved; nevertheless, the pace of enquiry remains brisk as new questions continue to arise and old ones are revisited. The growing interface between membrane traffic and other areas of cell biology — such as development, signalling, cytokinesis and the cytoskeleton - has contributed to the continued expansion of the field, while also attesting to its immediate relevance to allied topics in cell biology. As in other areas of cell biology, the advent of sophisticated imaging technology has opened up new avenues of study in membrane traffic, which will ensure that the field remains an engaging and vital area of cell biological enquiry for the coming decades. The aim of this collection is not to represent a comprehensive survey of the field, but rather to highlight a cross-section of emerging topics of interest such as regulated exocytosis and the generation of organelle identity.

We hope that this Focus will appeal not only to those directly involved in membrane traffic, but also to readers whose primary interest lies in other areas of cell biology. The Focus articles will be available free online for one month. In addition, these Focus articles will be available on a dedicated website (http://www.nature.com/ncb/webfocus/membrane), which also contains a continuously updated online archive of all relevant material published in Nature Cell Biology since the journal was launched five years ago.

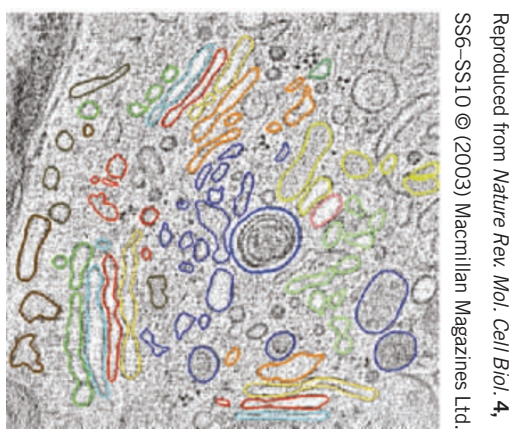

\section{Nothing to declare?}

We would like to draw your attention to an important piece of journal policy that has occasionally proved confusing: along with Nature and the other Nature research titles, Nature Cell Biology has for some time followed a policy of declaring 'competing financial interests' (CFIs).

The aim is to add transparency to the increasingly elaborate net of financial interests that pervades not only industrial and biotechnology research, but also academia — both at the institutional and personal level. It is not our aim to castigate research with a profitable bottom line - far from it. However, we believe that our readers are entitled to know of any significant financial interests of our authors in the work presented, so that they can form their own judgement of possible bias. A CFI declaration must not be viewed as the revelation of a dirty secret, nor as restating the obvious, but rather as a formal record of the financial context in which the research was carried out.

Our definition of financial interests is those that "through their potential influence on behaviour or content or from perception of such potential influences, could undermine the objectivity, integrity or perceived value of a publication" (this is further elaborated at http://www.nature.com/nature/submit/policies/competing/index.html). This definition encompasses the funding of research (including salaries, expenses and research tools), direct employment and, importantly, personal financial interests such as stock options, shares or patents. 'Competing' should be read as any financial interests that might be perceived as competing with the pure disinterested search for truth, wherever that search may lead.

We do not require a declaration of the monetary value of any interests; however, we do require declaration of all significant interests related to the scientific content of the article (our full policy statement details what constitutes 'significant'). Only financial interests directly relevant to the science covered in the article should be declared, but a direct industrial affiliation will usually qualify as a CFI. If in doubt, the key criterion is whether the authors have a specific prospect of financial gain or loss based on the outcome of the research.

The invitation to provide CFI information may be declined, but this fact will be published alongside the paper. We now apply this policy to both our primary research papers and review articles. Importantly, we also request that our referees inform us of any financial interests that may in principle influence their refereeing, and this is taken into account during referee selection.

We hope it is self-evident that the aim of this policy is not to denigrate application-oriented research; rather, it is to foster transparency, particularly at this exciting time of ever-increasing and ever-more intricate affiliations between academia and industry, and the increased level of public scrutiny this has precipitated (see, for example, Nature 429, 119 (2004) and Nature Neuroscience 6, 997 (2003)). If doubts remain, please consult our editors before publishing or agreeing to referee. 